\title{
Tomographic Characterization of Dislocations in Failure Regions of Broad Area InGaAs/AlGaAs Strained-Layer Single Quantum Well High Power Laser Diodes
}

\author{
Brendan Foran, Neil Ives, Terence Yeoh, Miles Brodie, Yongkun Sin, Nathan Presser,
} Maribeth Mason, and Steven C. Moss

The Aerospace Corporation, 2350 E. El Segundo Blvd., El Segundo, CA 90245-4691

In this paper we describe efforts to define the three-dimensional structure of dislocations found in the failure region of high power laser diodes which suffered catastrophic damage as a result of high-current operation in accelerated life-testing.

Broad area, multi-mode single emitter InGaAs/AlGaAs strained quantum-well laser diodes at 920-980 $\mathrm{nm}$ have become indispensable as pumps for fiber amplifiers used for long haul fiberoptic telecommunications [1]. Commercial diodes have advertised average mean time before failure (MTBF) as high as $>500$ khours (50 years). However, individual devices randomly fail catastrophically with known non-zero (and significant) statistics with no understood warning signs [2]. Randomly occurring catastrophic failure of semiconductor quantum well laser diodes has been attributed to thermal runaway associated with increase in non-radiative recombination occurring at extended dislocations arrays. Early transmission electron microscopy of failure sites in semiconductor laser diode materials showed that dislocation networks could propagate via a dislocation climb mechanism accelerated by recombination enhanced defect motion [3]. Understanding the complex three-dimensional (3D) structure of extended dislocation arrays is necessary to develop models of dislocation propagation associated with diode failure.

Traditional computed tomographic 3D reconstruction from a large tilt-series data set is problematic for dislocation arrays since diffraction contrast, used to image dislocations, has nonlinear intensities associated with special sample orientations. However, for special imaging conditions and sample orientations, dislocations may be simplified as discrete line sections bending through 3D space. This simplification of the sample-model allows 3D reconstruction by methods of discrete tomography [4]. Discrete tomography further simplifies analysis by allowing 3D reconstruction from a limited set of sample orientations as is more typically available for traditionally prepared samples with more broad areas of analysis.

This work employed annular dark field STEM with detector collection angles chosen to optimize diffraction contrast highlighting dislocations. Fig 3 outlines a graphical analog to discrete tomography applied to the dislocations shown in Figs 1 and 2. STEM images were propagated into $3 \mathrm{D}$ projections along the direction of the electron beam for a few sample orientations that showed high diffraction contrast. The 3D propagated images were then superimposed such that overlapping regions define a discrete model of the complex 3D structures of the dislocation arrays. Computational refinement of projection alignment and optimization of overlap were required to generate models relating device failure.

[1] Y. Sin, N. Presser, B. Foran, S. C. Moss, Proc. Of SPIE, 6876, (2008)

[2] V. Rossin, E. Zucker, M. Peters, M. Everett, and B. Acklin, Proc. of SPIE, 5336, 27 (2004).

[3] P. Petroff and R. L. Hartman, Applied Phys. Lett., 23, 469 (1973).

[4] S. Bals, K.J. Batenburg, et al., Nano Letters, 7(12), 3669 (2007). 

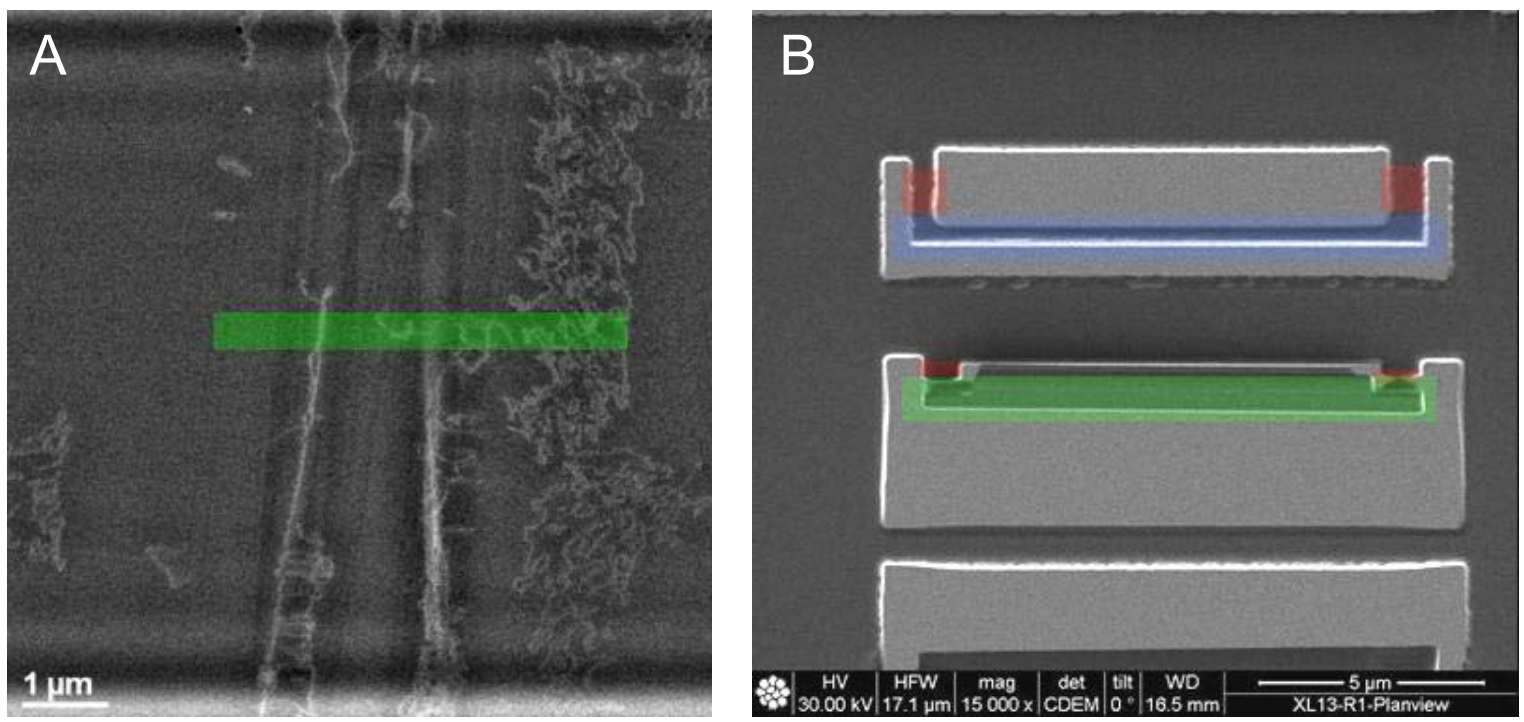

Figure 1. (A) Plan-view ADF STEM image showing contrast associated with dislocation arrays in the HPLD failure region. (B) SEM image from SEM-FIB instrument showing windows milled to allow ninety degree bending of sections prepared for cross-sectional imaging. Green region marks crosssectional sample employed for 3D characterization of dislocation arrays shown below.

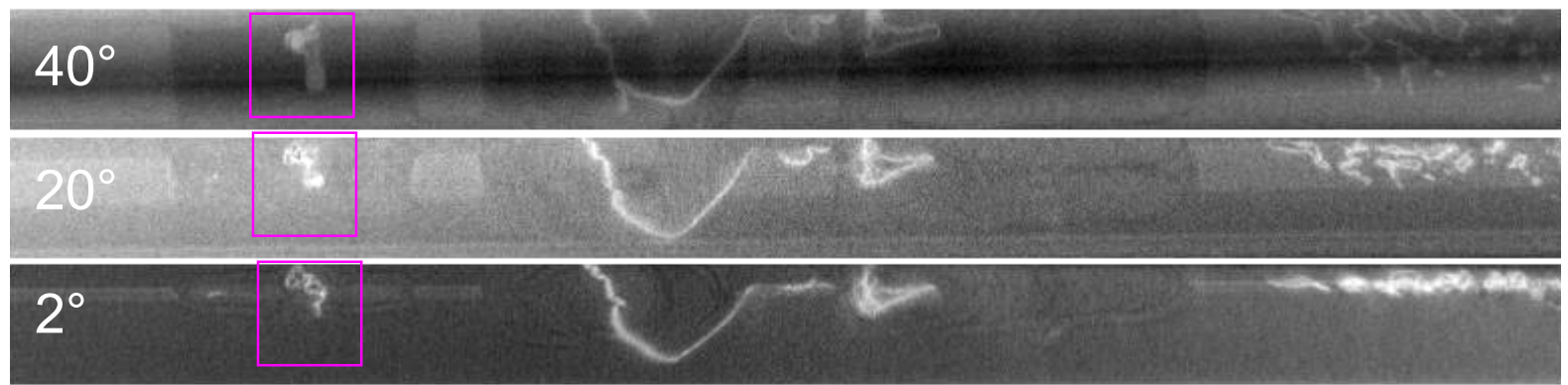

Figure 2. (a) ADF-STEM images recorded for the cross-sectional sample, marked by the green box in Fig 1 , imaged at three different sample orientations. The $2^{\circ}$ image was $\sim 2^{\circ}$ from the $<110>$ zone axis. The height of each image is 1 micron.
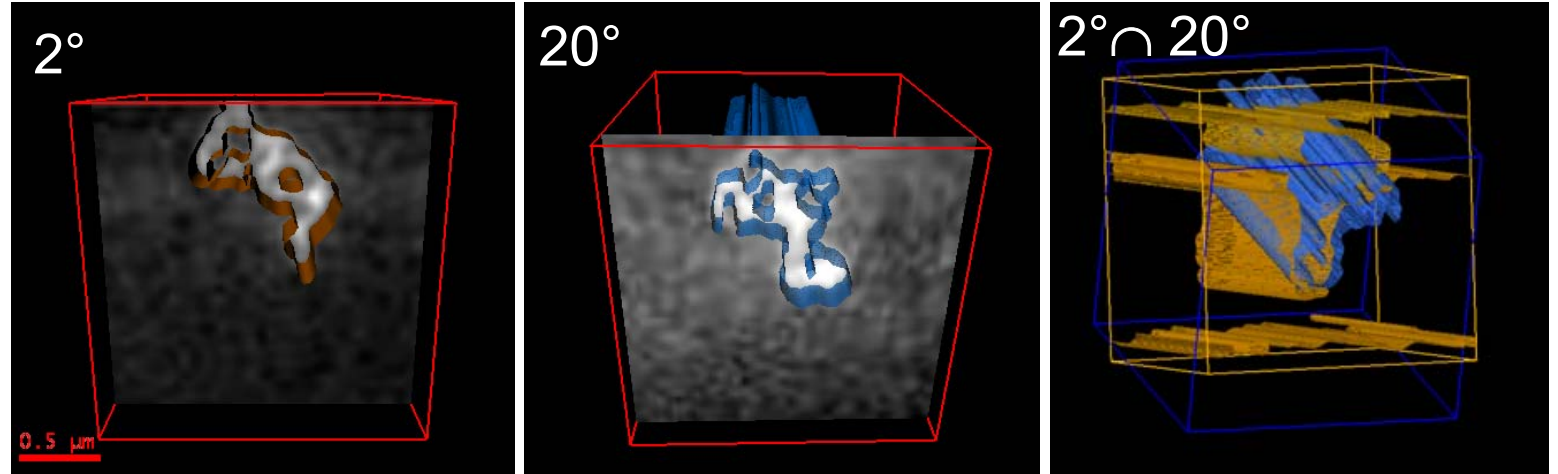

Figure 3. Propagated projection surfaces formed by repetition of individual ADF-STEM images for the dislocation marked by the purple box in Fig 2. The image at right shows the superposition of the two projected surfaces; the intersection of which defines a 3D model of the dislocation structure.

This work was supported under The Aerospace Corporation's Independent Research and Development Program 